\title{
Effect of Alumina Fibers on Fabrication Process and Characteristics of Alumina Fiber Reinforced Aluminum Alloy Composites
}

\author{
Masayuki Mizumoto ${ }^{1, a}$, Takeshi Ohgai ${ }^{1, b}$ and Akio Kagawa ${ }^{1, c}$ \\ ${ }^{1}$ Department of Materials Science and Engineering, Nagasaki University, 1-14 Bunkyo-machi, \\ Nagasaki 852-8521, JAPAN \\ amizumoto@nagasaki-u.ac.jp, bohgai@nagasaki-u.ac.jp, akagawa@nagasaki-u.ac.jp
}

Keywords: Fiber reinforced metal matrix composite, Pressure infiltration method, Alumina fiber.

\begin{abstract}
In order to develop the alumina fiber reinforcements optimized to FRMMCs, the effect of characteristics of alumina fibers on the fabrication process and the characteristics of the alumina fiber reinforced $\mathrm{Al}$ alloy composites was investigated. Alumina fibers which have different alumina content were prepared. Alumina content in the fibers was varied from $80 \%$ to $100 \%$. Al-4mass $\% \mathrm{Cu}$ alloy, Al-12mass\%Si alloy and Al-10masss\%Mg alloy were used as matrix. The FRMMC specimens were fabricated by a low-pressure infiltration process (LPI process). The formability of the preform was improved with increasing alumina content in the fibers. However, broken fibers were observed in the preform when alumina fibers with high alumina content were used. The number of the broken fibers seemed to be increased with increasing alumina content in the fibers. This result could be attributable to a change of fiber strength resulting from a change of alumina content in the fiber. The FRMMC specimens were characterized by using Vickers hardness test. The Vickers hardness of FRMMC specimens depended on the elasticity or the hardness of the fibers. The results obtained suggest that the characteristics of the FRMMCs largely depend on the intrinsic characteristics of the reinforcement fibers.
\end{abstract}

\section{Introduction}

Metal matrix composites (MMCs) are expected to be promising materials which can replace the existing metallic materials due to their superior mechanical and physical properties such as light weight, high thermal conductivity and high specific strength. Although there are many researches on the fabrication process and the characterization of various types of MMCs [1,2], most of the MMCs have been reported consist of a combination of commercially available reinforcements with matrix alloys. In order to develop MMCs which exhibit the excellent properties, it is essential to develop the reinforcements and matrix alloys optimized for an application to MMCs. However, there are few researches on the optimization of the reinforcements aiming for the application to MMCs. To develop the reinforcements optimized for MMCs, it is necessary to investigate the effect of the characteristics of the reinforcements on the fabrication process and characteristics of the MMCs in detail. In the present work, to develop the alumina fibers optimized to FRMMCs, the effect of characteristics of alumina fibers on the fabrication process and the characteristics of the alumina fiber reinforced $\mathrm{Al}$ alloy composites was investigated.

\section{Experimental Procedure}

In this work, alumina fibers (3-10 $\mu \mathrm{m}$ in diameter) which have different alumina content were prepared and were provided from DENKI KAGAKU KOGYO KABUSHIKI KAISHA. In order to investigate the effect of alumina content in the fibers on fabrication process and characteristics of the FRMMC specimens, alumina content in the fibers was varied from $80 \%$ to $100 \%$. Alumina content and general properties of the provided fibers are shown in Table 1. In general, with increasing alumina content in the fiber, fiber elasticity and strength are decreased. FRMMC specimens were fabricated by LPI process [3-6]. Al-4mass\%Cu alloy, Al-12mass\%Si alloy and Al-10masss\%Mg alloy were used as 
$\mathrm{Al}_{2} \mathrm{O}_{3} / \mathrm{SiO}_{2}(\%) \quad \alpha-\mathrm{Al}_{2} \mathrm{O}_{3}(\%) \quad$ Fiber elasticity Fiber strength

\begin{tabular}{lcccc}
\hline $80 \mathrm{~A}$ & $80 / 20$ & 0 & High & High \\
$95 \mathrm{~A}$ & $95 / 5$ & $\leqq 10$ & $\downarrow$ & \\
$97 \mathrm{AL}$ & $97 / 3$ & $1 \sim 14$ & & \\
$97 \mathrm{Am}$ & $97 / 3$ & $30 \sim 39$ & & $\downarrow$ \\
$97 \mathrm{AH}$ & $97 / 3$ & $50 \sim 59$ & $\downarrow$ & $\downarrow$ \\
$100 \mathrm{~A}$ & $100 / 0$ & $80 \sim 100$ & Low & Low \\
\hline
\end{tabular}

Table 1 Characteristics of alumina fibers with different alumina content.

matrix alloy. The volume fraction of the alumina fibers in the FRMMC specimens was varied from 7.5-12.5 vol\%. The pure aluminum particles (20 $\mu \mathrm{m}$ in diameter) were prepared to control the volume fraction of alumina fibers in FRMMC specimens. The mixture consisting of alumina fibers, the aluminum particles and organic binder was formed into the cylindrical shape as the preform by compression molding. Then the preform was fired at $773 \mathrm{~K}$ to remove the binder. The dimension of the preform was $\phi 15 \times 10 \mathrm{~mm}$. Then the preform was placed in the low-pressure infiltration apparatus shown in Fig. 1. Silica tube of $15 \mathrm{~mm}$ in diameter was used as a container that (b)
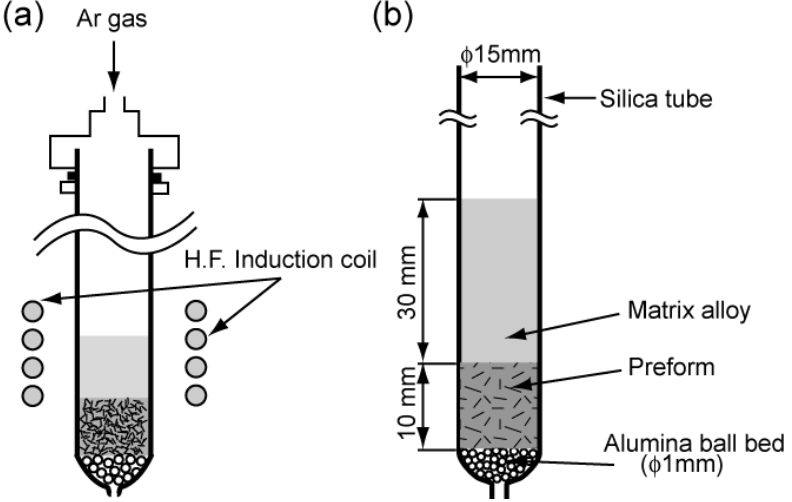

Fig. 1 Schematic drawing of (a) apparatus for low-pressure infiltration experiment, (b) sample set-up for $\mathrm{Al}_{2} \mathrm{O}_{3 \mathrm{f}} / \mathrm{Al}$ alloy composite。 had a small nozzle at the bottom to release the air inside the tube. Coarse alumina balls ( $1 \mathrm{~mm}$ in diameter) were laid at the bottom part of the silica tube as a bed layer, then the preform and matrix alloy were piled up in order in the silica tube. The upper matrix alloy was melted by H. F. induction heating, then the alloy melt was forced to infiltrate into the preform by applying an argon gas pressure on the melt surface. The infiltration pressure was 0.25 MPa. The microstructure was examined on the horizontal section with optical microscope and EDX-SEM. Vickers hardness of FRMMC specimens was measured using AKASHI AVK hardness tester with $98 \mathrm{~N}$ load in order to evaluate the effect of the fiber orientation and characteristics of alumina fibers on the mechanical properties of FRMMC specimens.

\section{Results and Discussion}

Effect of fiber strength on fabrication process of preform. Generally, in the fabrication process of the preform for fiber reinforced MMCs, it is required to compress the reinforcement fibers to form into the predetermined shape. It is considered that the condition of the fibers in the preform after compression molding would be influenced by fiber strength. Therefore it was investigated that the effect of fiber strength on the fabrication process of the preform and the condition of the fibers in the
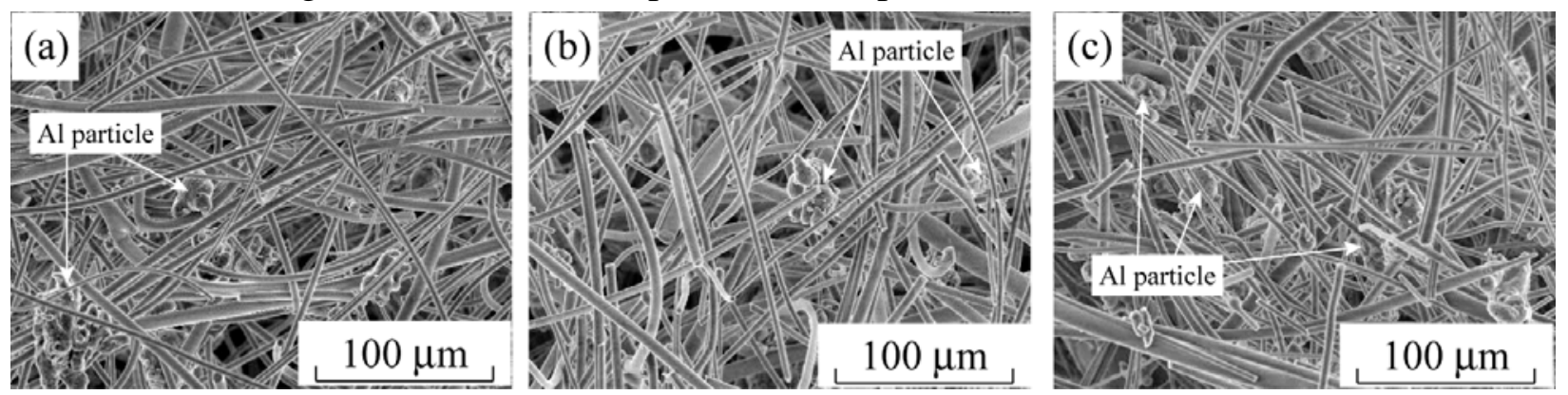

Fig. 2 SEM images of the preform consisting of pure aluminum particles and alumina fibers with different alumina content after compression molding. (a) $80 \mathrm{~A}$, (b) $97 \mathrm{~A}_{\mathrm{H}}$, (c) $100 \mathrm{~A}$. 
preform after compression molding. With decreasing alumina content in the fibers, the height of the preform after compression molding showed a tendency to increase in comparison with the height of the predetermined shape of the preform. Figure 2 shows the SEM images of the preform consisting of pure aluminum particles and alumina fibers with different alumina content after compression molding. When 80A fiber was used, few broken fibers were observed in the preform as shown in Fig. 2(a). On the other hand, when 100A fiber was used, many broken fibers were observed as shown in Fig. 2(c). The number of the broken fibers seemed to be increased with increasing alumina content in the fibers. These results can be attributable to a change of fiber strength resulting from a change of alumina content in the fiber. Schematic illustration of the deformation behavior of alumina fibers during compression molding is shown in Fig. 3. It is considered that alumina fibers in the preform would be subjected to local stress at contact points between alumina fibers and pure aluminum particles during compression molding. When alumina fibers which have high fiber strength, that is, high elasticity, are used, the fibers could be deformed elastically resulting in the formation of few broken fibers. On the other hand, when the fibers which have low fiber strength are used, the fibers would be broken easily at the contact points between alumina fibers and pure aluminum particles due to their low elasticity. It has been reported that a sufficient fiber length, 10 times longer than critical length, is required to reflect the characteristics of the reinforcement fibers in the mechanical properties of FRMMCs [1]. Thus these results suggest that alumina fibers which have high alumina content, that is, low fiber strength, are not suitable for the application for FRMMCs.

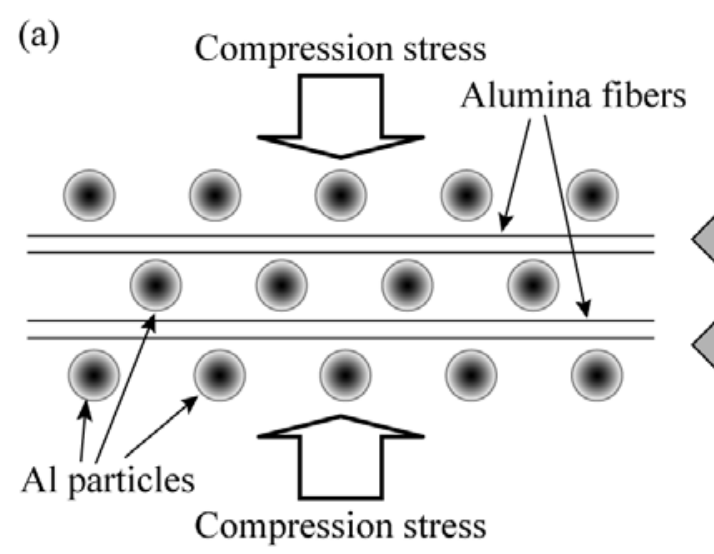

(b) Alumina fibers with low elasticity

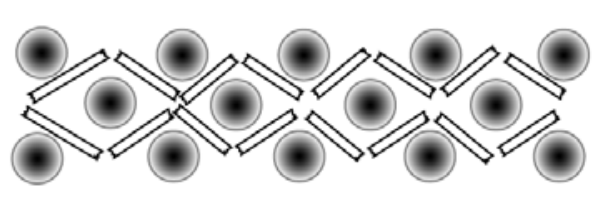

(c) Alumina fibers with high elasticity

Fig. 3 Schematic illustration of the deformation behavior of alumina fibers during compression molding. (a) Before compression molding, (b) Alumina fibers with low elasticity after compression molding and (c) Alumina fibers with high elasticity after compression molding.

Effect of matrix alloy composition on microstructure of FRMMCs. Figure 4 shows the microstructure of $10 \mathrm{vol} \%$ alumina fiber reinforced $\mathrm{Al}$ alloy composites with different matrix alloy. In each FRMMC specimens, alumina fibers seem to be distributed homogeneously and no casting defects are observed. Figure 5 shows the typical microstructure near the interface between $97 A_{L}$ fiber
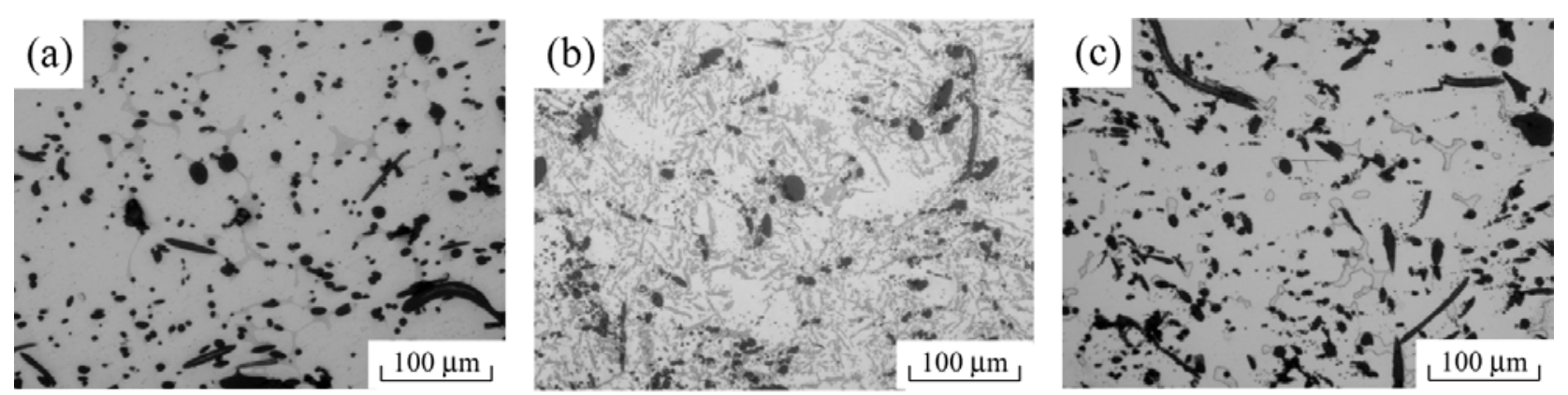

Fig. 4 Microstructure of $10 \mathrm{vol} \%$ alumina fiber reinforced $\mathrm{Al}$ alloy composites with different matrix alloy. (a) $\mathrm{Al}_{2} \mathrm{O}_{3 \mathrm{f}} / \mathrm{Al}-4$ mass\%Cu alloy composite, (b) $\mathrm{Al}_{2} \mathrm{O}_{3 \mathrm{f}} / \mathrm{Al}-12$ mass\%Si alloy compsite and (c) $\mathrm{Al}_{2} \mathrm{O}_{3 \mathrm{f}} / \mathrm{Al}-10 \mathrm{mass} \% \mathrm{Mg}$ alloy composite. 
and $\mathrm{Al}-\mathrm{Cu}, \mathrm{Al}-\mathrm{Si}$ and $\mathrm{Al}-\mathrm{Mg}$ alloys. In the case of $\mathrm{Al}-\mathrm{Cu}$ alloy and $\mathrm{Al}-\mathrm{Si}$ alloy composites, no reaction layer was observed at the interface. Although, in the case of Al-Mg alloy composite, no significant reaction layer was identified by SEM observation, small degradation of the fiber was observed as shown in Fig. 5(c). It has been reported that Al-Mg alloy melt reacts with alumina fibers, resulting in a degradation of the surface of alumina fibers [7]. However, the fiber degradation was so small that it would be expected not to affect the characteristics of FRMMCs fabricated by LPI process.
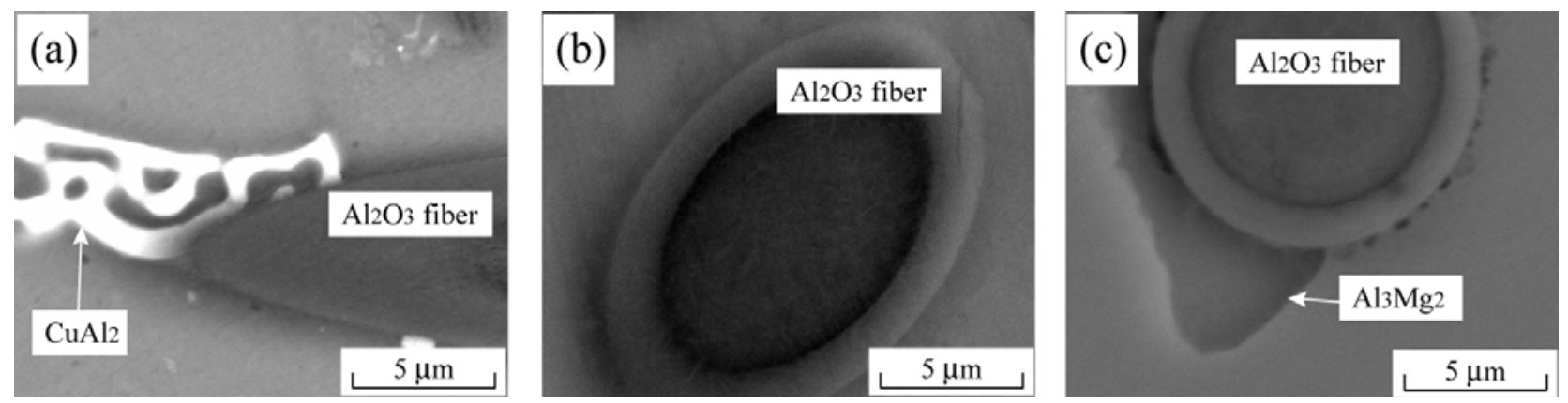

Fig. 5 Microstructure near the interface between alumina fiber $\left(97 \mathrm{~A}_{\mathrm{L}}\right)$ and various matrix alloy. (a) $\mathrm{Al}-4 \mathrm{mass} \% \mathrm{Cu}$ alloy matrix, (b) Al-12mass\%Si alloy matrix and (c) Al-10mass\%Mg alloy matrix.

Vickers hardness of alumina fiber reinforced MMCs. In order to evaluate the effect of fiber orientation and characteristics of alumina fibers on the mechanical properties of FRMMC specimens, Vickers hardness tests were carried out on the FRMMC specimens reinforced by various types of alumina fibers which have different mechanical properties as shown in Table 1 . The Vickers hardness was measured on two planes, parallel and perpendicular to the flow direction of the alloy melt during the infiltration process. The results of Vickers hardness test are shown in Fig. 6 in comparison with the hardness of the matrix alloy. The Vickers hardness of all FRMMC specimens was higher than that of matrix alloy. Present authors have reported the effect of metal particles introduced into the preform on the fiber distribution and the fiber orientation in the short fiber reinforced MMCs $[5,6]$. In the present work, the size of pure aluminum particles introduced into the preform was not large enough to orient the fibers parallel to the flow direction. Thus it was expected that the two-dimensional fiber orientation would be observed in the FRMMC specimens. As a result, it is considered that the Vickers hardness of the FRMMC specimens would vary depending on the plane that the hardness test was carried out. The Vickers hardness measured on the plane, perpendicular to the flow direction is higher than that measured on the plane, parallel to the flow direction in each specimen. This result indicates that most of the fibers in the FRMMC specimens fabricated in the present work were oriented perpendicularly to the flow direction. In addition, the Vickers hardness values of the FRMMC specimens varied according to the elasticity and hardness of the fibers. When the fibers which had high elasticity, for example $80 \mathrm{~A}$ or $95 \mathrm{~A}$, were used, the hardness value became higher due to the

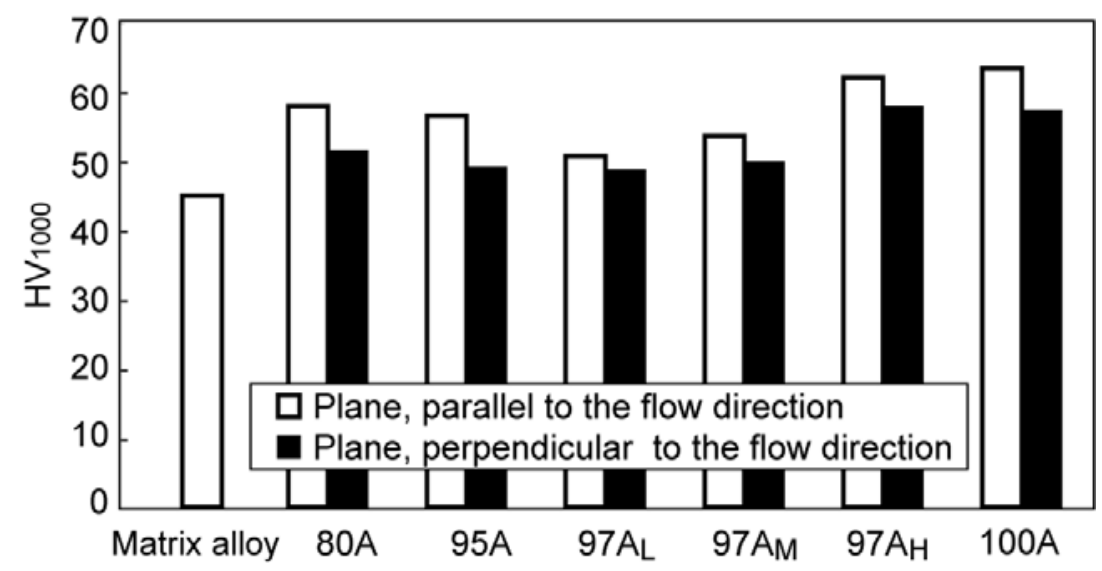

Fig. 6 Vickers hardness of $10 \mathrm{vol} \% \mathrm{Al}_{2} \mathrm{O}_{3 \mathrm{f}} / \mathrm{Al}$ mass\%Cu alloy compsoites mesured on the two planes, parallel and perpendicular to the flow direction during infiltration process. 
spring back effect resulted from excellent elasticity of the fibers. On the other hand, when the fibers which had low elasticity, for example $97 \mathrm{~A}_{\mathrm{H}}$ or $100 \mathrm{~A}$, were used, high intrinsic hardness of the fibers would contribute to an enhancement of the hardness of FRMMCs. From these results, it is suggested that the mechanical properties of FRMMCs would be strongly influenced by the mechanical properties of reinforcement fibers.

\section{Conclusions}

1. With increasing alumina content in the fibers, the formability of the preform was increased. However, the number of the broken fibers in the preform seemed to be increased with increasing alumina content in the fibers. It is considered that the improved elasticity of the alumina fibers resulted from the increment of the amount of $\mathrm{SiO}_{2}$ content in the fibers would prevent the fiber breakage during the compression molding.

2. No reaction layer was observed at the interface between alumina fibers and matrix in $10 \%$ vol\% alumina fiber/Al-4mass\%Cu alloy, Al-12mass\%Si alloy and Al-10massMg alloy composite fabricated by LPI process. A small degradation of the fiber was observed on the surface of alumina fibers in alumina fiber/ Al-10massMg alloy composite. However, the degradation was so small that it would not affect the characteristics of FRMMCs.

3. The Vickers hardness measured on the different two planes in FRMMC specimens indicated that most of the fibers in the FRMMC specimens were oriented perpendicularly to the infiltration direction. The Vickers hardness values of the FRMMC specimens varied according to the elasticity or hardness of the fibers. This result indicates that the mechanical properties of FRMMCs would be strongly influenced by the mechanical properties of reinforcement fibers.

\section{Acknowledgment}

This research was supported by the Ministry of Education, Culture, Sports, Science and Technology, Grant-in-Aid for Young Scientists (B), 20760507, 2008.

\section{References}

[1] Y. Nishida: Introduction to Metal Matrix Composites, Corona Publishing Corp., Tokyo, Japan (2001).

[2] D. Hull and T. W. Clyne: An Introduction to Composite Materials Second Edition, Syndicate of the Cambridge University Press, England (1996).

[3] M. Mizumoto, T. Murano and A. Kagawa: Materials Transactions, JIM Vol. 43 (2002), p. 2629.

[4] M. Mizumoto, Y. Tajima and A. Kagawa: Materials Transactions, JIM Vol. 45 (2004), p.1769.

[5] M. Mizumoto, Y. Kaneko and A. Kagawa: J. Japan Inst. Metals Vol. 68 (2004), p. 1047.

[6] M. Mizumoto, H. Ezaki and A. Kagawa: Material Science Forum Vol. 475-479 (2005), p. 2559.

[7] U. Rehman, S. Fox, H.M. Flower and D.R.F. West: J. Mater. Sci. 29 (1994), p. 1636. 